\title{
HIGH SPECTRAL AND TEMPORAL RESOLUTION OF PULSED EMISSIONS BY FOURIER TRANSFORM SPECTROMETRY
}

\author{
R. STRINGAT, G. FABRE, A.J. ROSS* and A. TOPOUZKHANIAN* \\ Laboratoire d'Optique Atomique et Moléculaire, Faculté des Sciences, Parc Valrose, \\ F-06034 Nice cedex, France \\ * Laboratoire de Spectrométrie Ionique et Moléculaire, Université Lyon I, 43 boulevard \\ du 11 novembre 1918, F-69622 Villeurbanne cedex, France
}

\begin{abstract}
By slaving a pulsed laser (a few $\mathrm{kHz}$ ) to a Fourier transform spectrometer of the "continuous scanning" type [BOMEM DA3], high resolution spectra of the pulsed emission can be obtained in the IR, visible and UV regions.

By inserting a fast gated integrator between the detector (PMT) and the amplifier in the sequence of data acquisition elements in the spectrometer, it is possible to record time resolved spectra for which the temporal resolution is imposed by the response time of the detector, typically $10-20 \mathrm{~ns}$.
\end{abstract}

The spectral intensity $B(\sigma)$ of the light source $-\sigma$ wavenumber- is the Fourier transform (inverse) of the interferogram $I(\delta)-\frac{1}{2} I(0)$ where $I(\delta)$ is the light intensity after the Michelson interferometer, as a function of the path difference $\delta ; B(\sigma)=\int_{-\infty}^{+\infty}\left[\mathrm{I}(\delta)-\frac{1}{2} \mathrm{I}(0)\right] \mathrm{e}^{-2 \pi i \sigma \delta} \mathrm{d} \delta$ (for a two-sided interferogram assuming that the reflectivities of mirrors are equal).

In our experiments, where the fluorescence is obtained with a pulsed laser, $I$ is also an explicit function of time: $I=I(\delta, t)$.

The detected electrical signal $S$ is the convolution of I with the impulsional response of the detection. Considering exclusively conditions where the variations of I with $t$ are slow compared to the response time of the entire detection, $S$ follows the variation of $I: S(\delta, t) \propto I(\delta, t)$.

The fonction $S$ is sampled at regular step of the path difference. 
The spectrometer we use (BOMEM DA3) is of the "continuous scanning" type i.e. that intensity measurements are performed during the flight of the moving mirror. The sampling steps available are $\lambda, \frac{\lambda}{2}, \frac{\lambda}{4}$ and $\frac{\lambda}{8}$ (where $\lambda$ is the wavelength of a reference He-Ne laser). Each sampling sequence begins with the generation of a trigger TTL sampling pulse deduced from the He-Ne interferogram and finishes with the $A / D$ conversion of $S$ at time $\Delta T$.

If we consider the case where the laser is slaved to the spectrometer $(1)$ by triggering it with delayed sampling pulses, the conversion is always realized at the same time $t$ ' of the fluorescence. The Fourier transform of $S\left(\delta, t^{\prime}\right)$ then gives the time-resolved spectrum $B\left(\sigma, t^{\prime}\right)$.

For visible and near I.R. fields, the apparatus is equipped with quantic detectors ( $\mathrm{Si}, \mathrm{InSb}$, In Ga As) associated with preamplifiers and amplifiers whose response time spreads from 10 to $20 \mu \mathrm{s}$. We can see that if the studied phenomena are slow compared to the tenth of $\mu \mathrm{s}$, one can easily obtain timeresolved spectra modifying $\Delta \mathrm{T}$. This technique has been previously applied by a few authors in the I.R. (2).

In order to extend the field of validity of the basic condition "rapid detection compared to the timevariation of the phenomenon" i.e. to be able to study faster phenomena we have to lower the time response of the detection.

The main part of the $10-20 \mu$ s comes from the preamplifier and the filter associated to the amplifier. To escape from these time limits, we used a fast gated Boxcar integrator (2ns time response) connected directly to the detector.

The Boxcar integrates the input signal during the gate. The "last-sample" output from the integrator is then normalized by the gate width to provide a constant voltage which is proportional to the average of the input signal during the sampling gate. This constant signal is then injected into the spectrometer amplifier input.

To cover the visible and UV regions, the Boxcar was associated to a PMT (EMI 9558 QB) whose f.w.h.m. of the anode pulse was about $20 \mathrm{~ns}$.

The sampling pulses (frequency $6.32 \mathrm{kHz}$ ) are used to trigger the copper vapour laser (Oxford Lasers CU 25) after a chosen delay. The laser pulse occurs a very short time later.

The signal from the photomultiplier is sampled by a Stanford Research Systems Boxcar integrator (SR 250), using a gate $\tau=20 \mathrm{~ns}$ wide opening after a delay $t^{\prime}$ beyond the laser pulse starting. The 
integrated signal is then introduced into the spectrometer amplifier and digitized at time $\Delta \mathrm{T}$. The value for $\Delta \mathrm{T}$ is programmed on the host computer. Because the signal from "last sample" is constant, the actual time at which conversion occurs is not important, provided that all the steps have been performed and that the amplified "last sample" signal has reached its stable value.

Our sole aim being to demonstrate the possibility of doing time resolved spectroscopy with this system, we chose an easily prepared source, iodine in the gas phase, as a test case. The B-X system of $\mathrm{I}_{2}$ is excited by the yellow line $(578 \mathrm{~nm}$ ) of the copper vapour laser (pulse duration about $50 \mathrm{~ns}$ ).

The yellow laser line of the copper vapour laser has a frequency which is quasi-resonant with three transitions in the $\mathrm{BO}_{\mathrm{u}}^{+}-\mathrm{X} 0_{\mathrm{g}}^{+}$system of $\mathrm{I}_{2}$. We observe fluorescence from three levels of the $\mathrm{BO}_{\mathrm{u}}^{+}$ state: $v^{\prime}=14, v^{\prime}=16$ and $v^{\prime}=20$, which are not predissociated.

The figure shows part of the fluorescence spectrum recorded at a resolution of $1.5 \mathrm{~cm}^{-1}$ at different times. The resolution and signal/noise ratio selected are sufficient to be able to follow the emission from levels pumped directly by the laser.

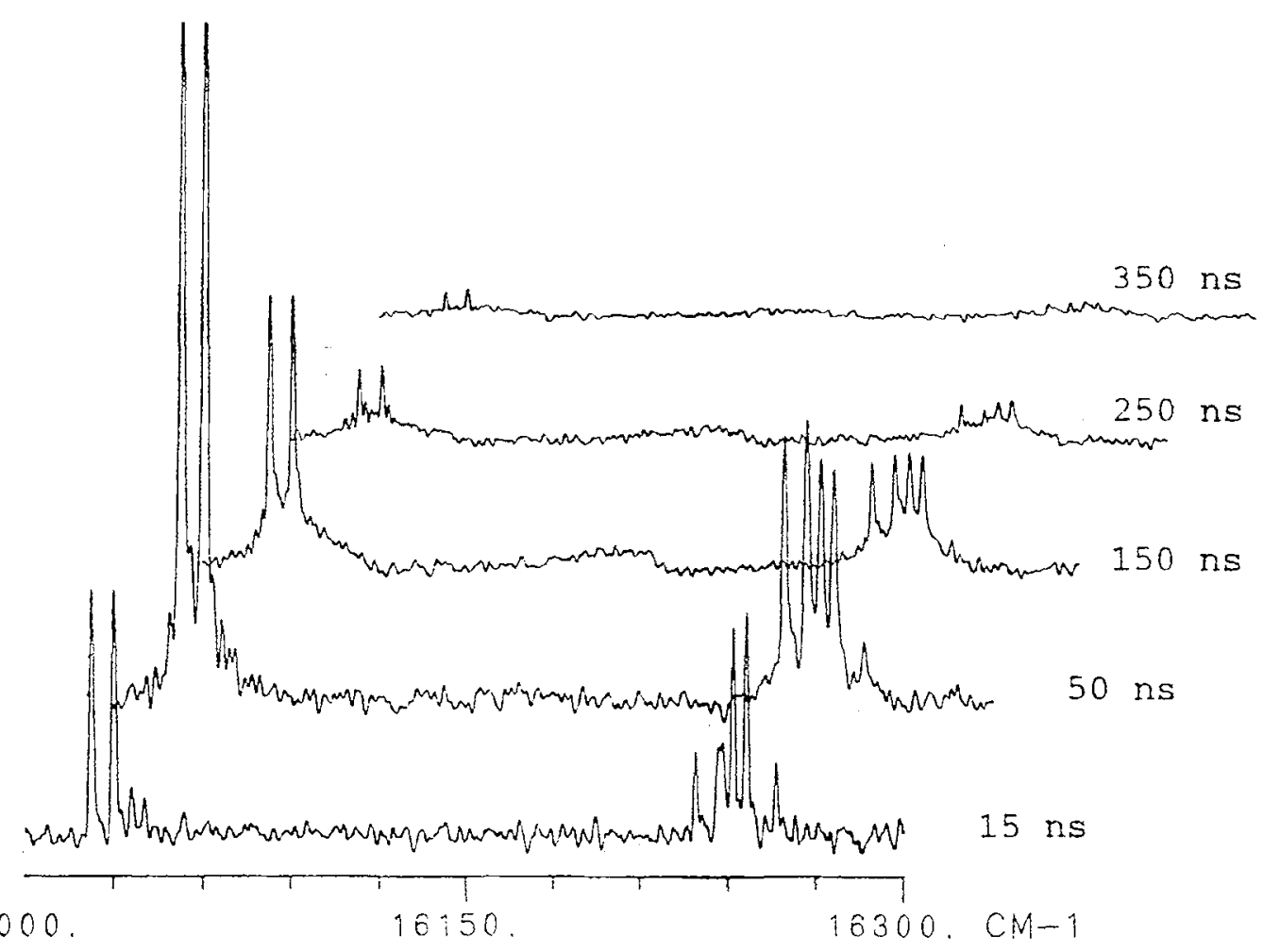


The lifetime deduced are $\tau_{16}=104 \pm 5 \mathrm{~ns}$ and $\tau_{14}=92 \pm 4 \mathrm{~ns}$.

The radiative lifetimes of the vibrational levels of the $B$ state can be calculated from extensive data given in the thesis of M. BROYER(3), and assuming that collisions with iodine are the only cause of decrease in the lifetime of these levels, we can predict effective lifetimes of these levels at room temperature assuming that the value of $\tau$ is not $J$ dependent as found in the work of M. BROYER.

At $25^{\circ} \mathrm{C}$, the vapour pressure of iodine is around $300 \mathrm{mTorr}$, and the lifetime of the vibrational levels of the $B$ state observed here are of $196(11) n s$ for $v^{\prime}=16$ and $192(9) n s$ for $v^{\prime}=14$. Residual impurities in our iodine cell are the most likely explanation for the much shorter value of $\tau$ found in this work, the cell was originally made for calibration purposes, not for lifetime measurements.

(1) STRINGAT, R, FABRE, G, CIRIO, L and ROSS, A, Optics Comm. 81, 3-4 (1991), 179

(2) MANTZ, A.W., Appl. Optics 17 (1978) 1347

BURCHELL, D.J., LASCH, J.E., FARRIS, R.J. and HSU, S.L., Appl. Optics 23 (1982) 965

AKER, P.M. and SLOAN, J.J., J. Chem. Phys. 85 (1986) 1412

DONALDSON, D.J. and LEONE, S.R., Chem. Phys. Letters 132 (1986) 240

KRUUS, E.T., NIEFER, B.I. and SLOAN J.J., J. Chem. Phys. 88 (1988) 985

FLETCHER, T.R.and LEONE, S.R., J. Chem. Phys. 88 (1988) 4720

(3) BROYER, M, Thesis University Paris VI (1977) 